\title{
Column 9 \\ The Power of Dialogical Tools \\ in Participatory Learning
}

\author{
Akihiko Kotera
}

\section{How Can We Promote Dialogue?}

It seems that most of the complex problems in the world are made difficult to solve by a lack of dialogue. Needless to say, "dialogue" does not immediately solve everything, but at least we can find much more hope compared with a situation without dialogue.

Participatory learning is one method for acquiring new knowledge and skills (Fig. C9.1). It is a method that emphasizes the importance of encouraging participants to take spontaneous actions such as experiencing and interacting and to learn from the process by themselves. In reality, however, it is quite difficult to establish a rich dialogue between participants and the facilitators of participatory learning.

One of the factors that inhibit dialogue in participatory learning is the means of communication of scientific knowledge and information. Various devices and tools, such as geographic information maps, illustrations, and models, have been used to convey information in an easy-to-understand manner. However, in many cases, the very tools that are supposed to provide useful information unintentionally interfere with the dialogue with the participants because their expertise is not translated well. This is because the act of reading and understanding geographical maps and graphs correctly is a specialized skill that requires a certain amount of training. In addition, most tools are designed for one-way transmission of information and have not been designed to be used in dialogue. In this context, new information communication tools/interfaces such as "Tangible Bit" are expected to be utilized.

\footnotetext{
A. Kotera $(\bowtie)$

Global and Local Environment Co-creation Institute, Ibaraki University, Ibaraki, Japan

e-mail: akihiko.kotera.moctuyen@vc.ibaraki.ac.jp
} 
Fig. C9.1 Scene of participatory learning
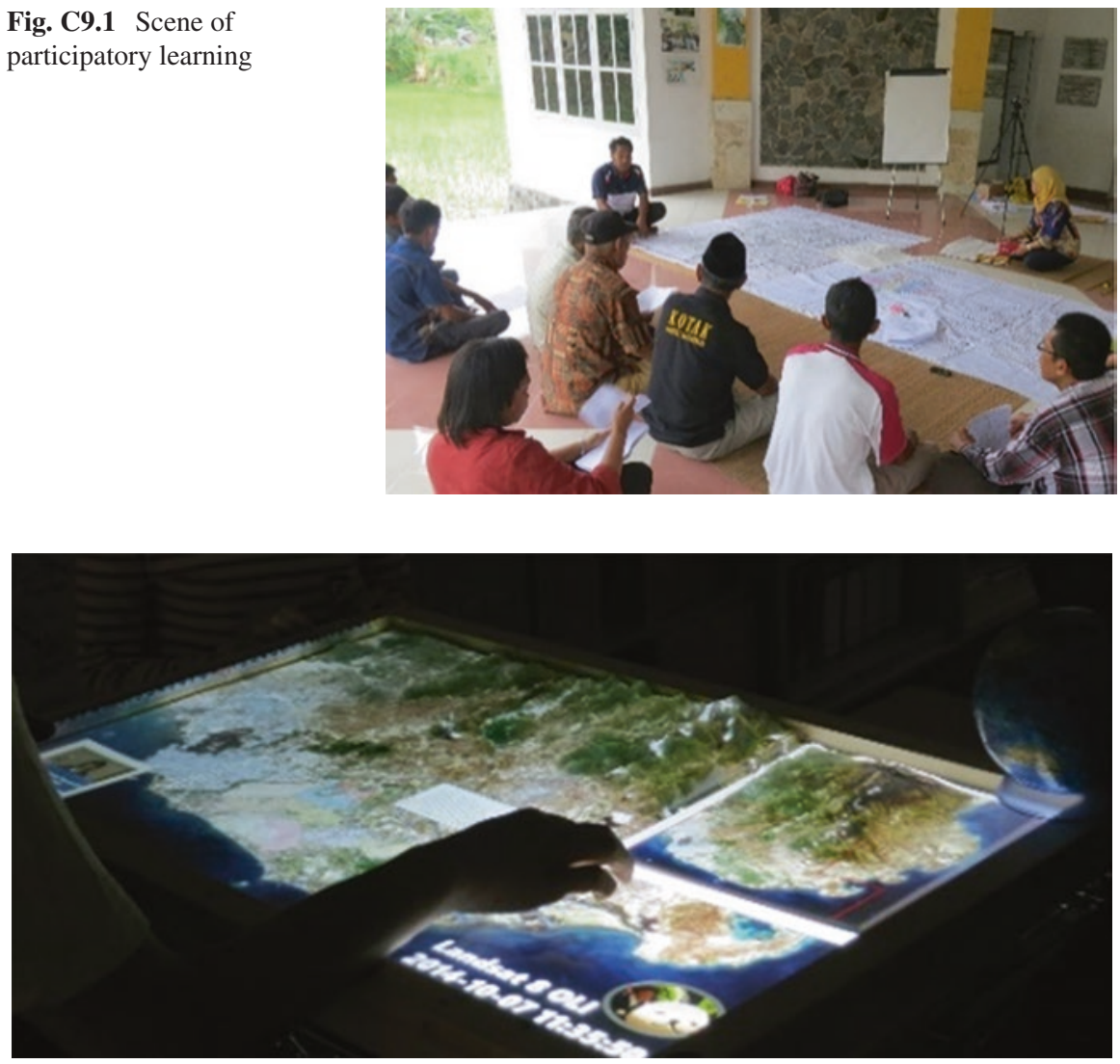

Fig. C9.2 Appearance of the tangible GIS

\section{Designing the Tool}

"Tangible Bit" consists of a 1:7500 scale three-dimensional (3D) terrain screen, a projector, a tablet PC, and an infrared camera-based touch sensor, and can project various information on the 3D terrain of the target area (Fig. C9.2). This system can be assembled and moved easily in the field. The table-top style allows participants to surround the 3D terrain screen naturally at a close distance from each other without creating physical barriers to access and interact with the information. The information contents to be displayed include satellite remote sensing images, various GIS thematic maps such as land use maps, water management organization charts, 
videos, and scanned images of existing paper maps, which are necessary and easy to learn. Each image has been geometrically corrected to fit the undulations of the 3D screen.

By superimposing these contents as needed and projecting them on the $3 \mathrm{D}$ topographic screen, unlike conventional flat maps, users can intuitively grasp various contents as well as topographic and hydrological information such as mountain slopes, height differences, and water flow directions. Furthermore, by touching and sliding your finger on the surface of the 3D screen, users can sense the information intuitively.

\section{Trying It Out}

The tool has been used in the field of collaborative water resources management with community participation in the Bilibili Irrigation command in southern Sulawesi and the Saba River Basin in northern Bali, Indonesia (Kubota 2016). The opportunities for using this tool include water user meetings, community stakeholder meetings, and meetings at universities and public institutions, and the information content used depends on the purpose of the meeting.

These meetings are held periodically by water users, where, for example, water allocation plans for irrigation in rice transplanting season are discussed. As a basis for discussing more rational water allocation, we analyzed the need to increase the common understanding of water resources in the entire region. The goal of the study was to understand (1) where and how much water used in the paddy fields is generated and how it flows, and (2) how the topography and environmental conditions of the paddy fields are distributed, both at the watershed scale and at the scale of each individual paddy field.

\section{What Kind of Changes Did the New Tool Bring?}

Although we have conducted similar learning using paper-based media in the past, the major change that we observed using this device was the atmosphere of the place, which clearly led to meaningful and prolonged "dialogue" (Fig. C9.3). Even members who do not usually speak up began to participate actively in the dialogue. This was triggered by the moment when each participant, including us, felt that they could intuitively understand the information (i.e., happy), and at the same time, were convinced that they were sharing the same feeling (i.e., fun). 

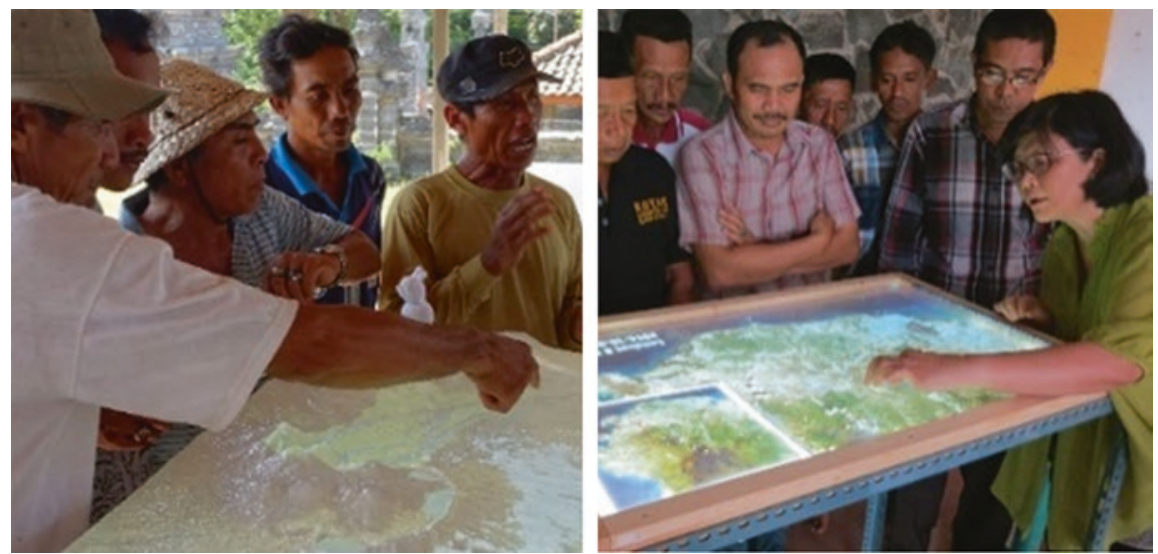

Fig. C9.3 Scenes of participatory learning and mutual dialogue with the tangible GIS

\section{What Have We Learned as Scientists?}

It was also necessary for scientists to change our way of talking and our attitude toward dialogue to master the new tools. What we later realized was that the most significant change for us was also that participatory learning felt more fun than ever. The fun was in the serious dialogue with the participants, and we believe that the same feeling was conveyed and shared by the participants. At the same time, the tools provided information that was easy to understand, and also had a synergistic effect on the dialogue among all the participants, including us. This is the power of dialogical tools.

\section{Reference}

Kubota J (ed) (2016) Sharing the water resources. Bensei shuppan, Tokyo. (in Japanese)

Open Access This chapter is licensed under the terms of the Creative Commons Attribution 4.0 International License (http://creativecommons.org/licenses/by/4.0/), which permits use, sharing, adaptation, distribution and reproduction in any medium or format, as long as you give appropriate credit to the original author(s) and the source, provide a link to the Creative Commons license and indicate if changes were made.

The images or other third party material in this chapter are included in the chapter's Creative Commons license, unless indicated otherwise in a credit line to the material. If material is not included in the chapter's Creative Commons license and your intended use is not permitted by statutory regulation or exceeds the permitted use, you will need to obtain permission directly from the copyright holder.

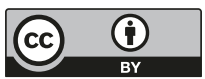

